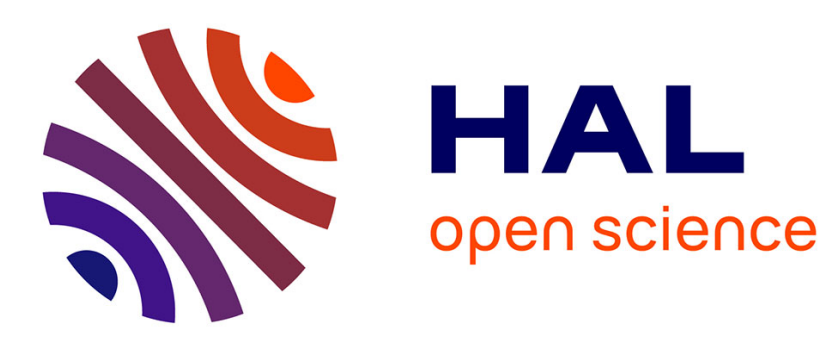

\title{
16. Étude des structures subharmoniques par effet tunnel entre supraconducteurs
}

\author{
I. Giaever, H.R. Zeller
}

\section{To cite this version:}

I. Giaever, H.R. Zeller. 16. Étude des structures subharmoniques par effet tunnel entre supraconducteurs. Revue de Physique Appliquée, 1970, 5 (6), pp.905-905. 10.1051/rphysap:0197000506090501. jpa-00243486

\section{HAL Id: jpa-00243486 https://hal.science/jpa-00243486}

Submitted on 1 Jan 1970

HAL is a multi-disciplinary open access archive for the deposit and dissemination of scientific research documents, whether they are published or not. The documents may come from teaching and research institutions in France or abroad, or from public or private research centers.
L'archive ouverte pluridisciplinaire HAL, est destinée au dépôt et à la diffusion de documents scientifiques de niveau recherche, publiés ou non, émanant des établissements d'enseignement et de recherche français ou étrangers, des laboratoires publics ou privés. 


\title{
16. ÉTUDE DES STRUCTURES SUBHARMONIQUES PAR EFFET TUNNEL ENTRE SUPRACONDUCTEURS
}

\author{
I. GIAEVER et H. R. ZELLER
}

General Physics Laboratory, Schenectady, New York

La caractéristique courant-potentiel obtenue lors d'une expérience d'effet tunnel entre deux supraconducteurs identiques comporte souvent des structures pour des énergies $2 \Delta / n$ où $2 \Delta$ est l'énergie de la bande interdite et $n$ est un nombre entier. L'origine de ces structures subharmoniques n'a jamais été établie correctement et bien souvent elles ont été reliées à un effet Josephson.

Nous avons étudié expérimentalement ces structures subharmoniques et nous obtenons trois nouveaux résultats: (1) l'amplitude de la structure peut être augmentée proportionnellement au carré de l'élément de matrice tunnel; (2) la structure est très sensible à l'application d'un rayonnement micro-onde externe ; (3) quand on utilise deux supraconducteurs différents $a$ et $b$, les structures apparaissent pour des énergies $2 \Delta_{\mathrm{a}} / 2 n, 2 \Delta_{\mathrm{b}} / 2 n$ et $\left(\Delta_{\mathrm{a}}+\Delta_{\mathrm{b}}\right) / 2 n+1$.

Les résultats expérimentaux laissent fortement supposer que les structures sont liées à une autodétection d'émission Josephson. Pour expliquer l'amplitude des structures, il est nécessaire de supposer que l'élément de matrice tunnel est si grand qu'il n'est pas possible de linéariser les équations Josephson. 